\title{
Determination of Urinary Pyrraline by Solid-Phase Extraction and High Performance Liquid Chromatography
}

\author{
Kazuhiro Yoshinara, ${ }^{a}$ Reiko Kiyonami, ${ }^{b}$ Yumi Shimizu, ${ }^{a}$ and Masatoshi Beppu*,a \\ Laboratory of Public Health, School of Pharmacy, Tokyo University of Pharmacy and Life Science, ${ }^{a}$ 1432-1 Horinouchi,

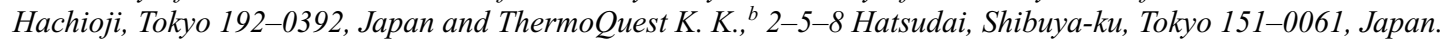 \\ Received February 20, 2001; accepted April 18, 2001
}

\begin{abstract}
Pyrraline is one of the advanced glycation end products formed under non-enzymatic and non-oxidative conditions in vivo. In this study, we developed a novel method for determination of urinary pyrraline using solidphase extraction as a pretreatment procedure prior to determination by high performance liquid chromatography (HPLC). The Oasis ${ }^{\text {TM }}$ HLB solid-phase extraction cartridge was used for pretreatment of urine samples without hydrolysis. The chromatogram obtained clearly revealed the peak for urinary pyrraline owing to prior removal of interfering substances in urine samples. The recovery rate of pyrraline was $97.2 \pm 3.3 \%(n=6)$. The mean excretion level of urinary pyrraline in healthy control $(20-77$ years old, $n=30)$ was $1.42 \pm 0.65 \mu \mathrm{mol} / \mathrm{mmol}$ creatinine, and the daily variation in the excretion level was considered to be insignificant. We propose the above procedure as a simple, rapid, and accurate method for determination of pyrraline levels in urine.
\end{abstract}

Key words advanced glycation end product; pyrraline; HPLC; solid-phase extraction; urine; atmospheric pressure chemical ionization tandem mass spectrometry

Long-lived proteins such as collagen, are partially or wholly modified in their molecular structure by glycation, resulting in accumulation of aminoketoses and formation of advanced Maillard reaction products, also known as advanced glycation end products (AGEs). Pyrraline, one of the major AGEs, is formed via a non-enzymatic reaction between reducing sugar and the epsilon-amino group of lysine residues of proteins, ${ }^{1)}$ and is thought to cause damage to tissues. ${ }^{2)}$ AGE formation pathways are believed to consist of two types; one requires oxidative conditions (glycoxidation) and the other non-oxidative conditions. Pentosidine is a product of the former pathway ${ }^{3-6)}$ and pyrraline is a product of the latter pathway. ${ }^{7)}$

A previous study demonstrated by immunological methods that the pyrraline level is increased in diabetic sclerosed arterial and extracellular matrix, as well as in the sclerosed matrix of glomeruli. ${ }^{8}$ Another study also demonstrated by electrospray ionization (ESI) mass spectrometric detection an increase in pyrraline level of the plasma from uremic subjects. ${ }^{9)}$ The level of plasma 3-deoxyglucosone, ${ }^{10,11)}$ an intermediate product in pyrraline and pentosidine formation, has been reported to increase in diabetic ${ }^{12,13)}$ and in uremic individuals. $^{14)}$

Recently, chromatographic methods were applied in the measurement of the urinary level of pyrraline, and its increase in diabetic patients was demonstrated, ${ }^{15,16)}$ suggesting usefulness of the methods for evaluation of diabetic complications. However, there are few other reports describing chromatographic techniques for determination of pyrraline levels in biological fluids. In this paper, we describe development of a rapid and accurate method for the determination of urinary pyrraline using solid-phase extraction prior to separation and analysis by HPLC. Moreover, the proposed method was applied to analysis of urine samples obtained from healthy volunteers.

\section{MATERIALS AND METHODS}

Chemicals L-Lysine, D-glucose, acetonitrile (HPLC grade) and trifluoroacetic acid (for amino acid sequencing analysis; TFA) were purchased from Wako Pure Chemical Ind., Ltd. (Tokyo, Japan) and used without further purification. The solid-phase extraction cartridge, Oasis ${ }^{\mathrm{TM}}$ HLB ( $3 \mathrm{ml}$, Waters Co., MA, U.S.A.), was used for pretreatment of urine samples. Other reagents obtained from commercial suppliers were of special reagent grade and/or analytical grade.

Preparation of Pyrraline Standard Standard pyrraline was synthesized and purified, with some modifications, according to the method of Nakayama et al. ${ }^{1)}$ L-Lysine $(6.60 \mathrm{~g})$ and D-glucose $(8.10 \mathrm{~g})$ were dissolved in $100 \mathrm{ml}$ of $0.5 \mathrm{M}$ sodium phosphate buffer ( $\mathrm{pH} 8.5$ ). The mixture was maintained at $80^{\circ} \mathrm{C}$ for $2 \mathrm{~d}$, and then cooled. The cooled reactant was loaded onto a gel-filtration column $(50 \times 1000 \mathrm{~mm}$, Bio Gel P-2, 45-90 $\mu \mathrm{m}$, Bio-Rad Labs., CA, U.S.A.) using 10\% $(\mathrm{w} / \mathrm{v})$ acetic acid as the elution solvent at a flow rate of 36 $\mathrm{ml} / \mathrm{h}$. The absorbance at $298 \mathrm{~nm}$ of each fraction $(16 \mathrm{ml} /$ tube $)$ was determined, and the fractions containing pyrraline were mixed together and evaporated to dryness. The HPLC eluent was added, and then the preparative HPLC procedure was performed. Briefly, a preparative ODS column (Wakosil-II 5C18 AR, $10 \mathrm{~mm} \times 250 \mathrm{~mm}$, Wako Pure Chemical Ind., Ltd., Japan) was used with the flow rate maintained at $4.5 \mathrm{ml} / \mathrm{min}$. Other conditions were the same as in the analytical method described below. The standard pyrraline obtained was analyzed by mass spectrometry and NMR spectrometry to confirm its structure.

Pretreatment of Samples The cartridge for pretreatment was used as follows. The cartridge was preconditioned with $3 \mathrm{ml}$ of methanol, and equilibrated with $3 \mathrm{ml}$ of water before loading the sample. One milliliter of urine sample was then applied to the cartridge, followed by washing the cartridge with $2 \mathrm{ml}$ of water. Finally, pyrraline was eluted from the cartridge with $3 \mathrm{ml}$ of acetonitrile containing $1.0 \mathrm{ml} / 1$ of TFA, and the eluent was evaporated to dryness under reduced pressure. The dried residue was then dissolved in $1 \mathrm{ml}$ of HPLC eluent described below and an aliquot $(10 \mu \mathrm{l})$ of each sample was applied to the analytical HPLC system.

Chromatographic Procedure and Instruments Sepa- 
ration and determination of pyrraline concentration in a urine sample were carried out by HPLC. The HPLC system was equipped with a L-6200 solvent delivery pump (Hitachi Co., Japan), a model L-4200 UV-VIS detector (Hitachi Co., Japan) set at $298 \mathrm{~nm}$, and a Wakopak column (Wakosil-II 5C18 AR, $4.6 \times 250 \mathrm{~mm}$, Wako Pure Chemical Ind., Ltd., Japan). The HPLC eluent consisted of $8 \%$ acetonitrile $(\mathrm{v} / \mathrm{v})$ containing $1.0 \mathrm{ml} / 1$ of TFA. The flow rate was maintained at $0.8 \mathrm{ml} / \mathrm{min}$ and the column was kept at $40{ }^{\circ} \mathrm{C}$. The peak area was calculated by using a model D-2500 automatic integrator (Hitachi Co., Japan). The total cycle time between two injections was $50 \mathrm{~min}$ including column washing with acetonitrile containing $1.0 \mathrm{ml} / 1$ of TFA.

In order to identify the urinary pyrraline, HPLC coupled with quadrupole ion trap multiple mass $\left(\mathrm{MS}^{\mathrm{n}}\right)$ spectrometer (LCQ DECA ion trap mass spectrometer, ThermoQuest, San Jose, CA, U.S.A.) equipped with atmospheric pressure chemical ionization (APCI) source was used. The APCI vaporizer was operated at $450{ }^{\circ} \mathrm{C}$, with a heated capillary temperature of $250^{\circ} \mathrm{C}$. The sheath gas and auxiliary gas flow rate was maintained at 85 and 10 of the arbitrary unit, respectively. The source current was set to $5 \mu \mathrm{A}$ and the mass spectra were acquired in positive ion tandem mass spectrometry (MS/MS) full scan mode.

Control Subjects Control urine samples were obtained from 30 healthy volunteers aged $22-70$ years old. None of the subjects was taking any medication or had any recognizable disease or history of renal disease or diabetes mellitus. Spot urine samples were collected between 9 and 10 a.m. without dietary restrictions and stored at $-20{ }^{\circ} \mathrm{C}$ until analysis.

\section{RESULTS AND DISCUSSION}

Suitable Pretreatment Conditions Removal of substances that interfere with the chromatographic determination of pyrraline levels in urine samples is necessary for obtaining reliable results. The pretreatment of urine samples using the Oasis ${ }^{\mathrm{TM}}$ HLB cartridge consists of the following three steps: sample loading, washing, and elution of pyrraline from the cartridge. When the sample was loaded and passed through the cartridge, urinary pyrraline was trapped in the cartridge while other interfering substances flowed out. The cartridge was then washed with water. Figure 1A shows the relationship between the volume of water used as the washing solvent and the quantity of urinary pyrraline retained in the cartridge. When washing solvent less than $2 \mathrm{ml}$ was used for washing the cartridge, pyrraline was completely retained, while increasing the volume to more than $3 \mathrm{ml}$ resulted in a gradual increase in pyrraline elution. After washing, pyrraline was eluted with acetonitrile containing $1.0 \mathrm{ml} / 1$ of TFA as an elution solvent. Elution with $3 \mathrm{ml}$ or more of the elution solvent resulted in complete extraction of pyrraline as shown in Fig. 1B. Based on these results, we decided to use $2 \mathrm{ml}$ of washing solvent and $3 \mathrm{ml}$ of elution solvent.

Chromatogram for Urinary Pyrraline Typical chromatograms for pyrraline extracted by the proposed method are shown in Fig. 2. Standard and urinary pyrraline are shown as a single and sharp peak with the same retention
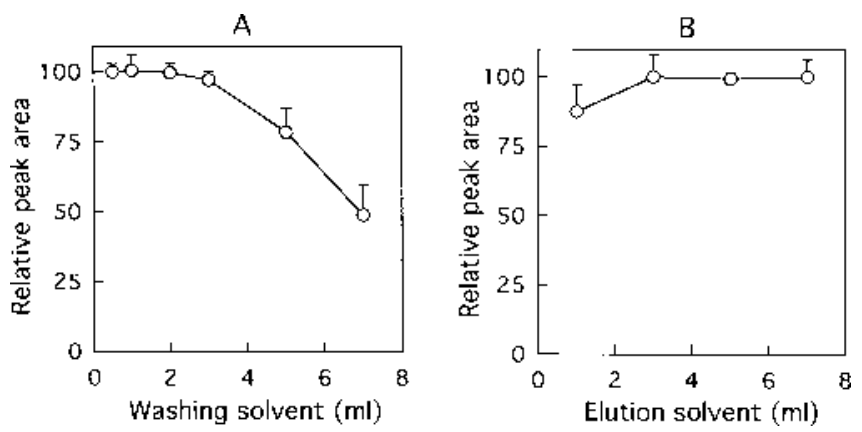

Fig. 1. Relationship between the Volume of Washing Solvent and Recovery of Pyrraline (A), and between the Volume of Elution Solvent and Recovery of Pyrraline From the Cartridge for Pretreatment (B)

For each point in (A), urine samples from 5 healthy volunteers were analyzed. After sample loading, various volumes of the washing solvent were passed through the cartridge and then pyrraline was extracted by eluting $3 \mathrm{ml}$ of the elution solvent. The recovery obtained using the least volume of the washing solvent $(0.5 \mathrm{ml})$ was taken as 100 , and the data were expressed as relative peak area. As for (B), after urine sample was loaded onto the cartridge for pretreatment, the cartridge was washed with $2 \mathrm{ml}$ of the washing solvent, and then pyrraline was extracted using various volumes of the elution solvent. The recovery obtained using $7 \mathrm{ml}$ of elution solvent was taken as 100 , and the data were expressed as relative peak area. Other conditions were the same as in (A).

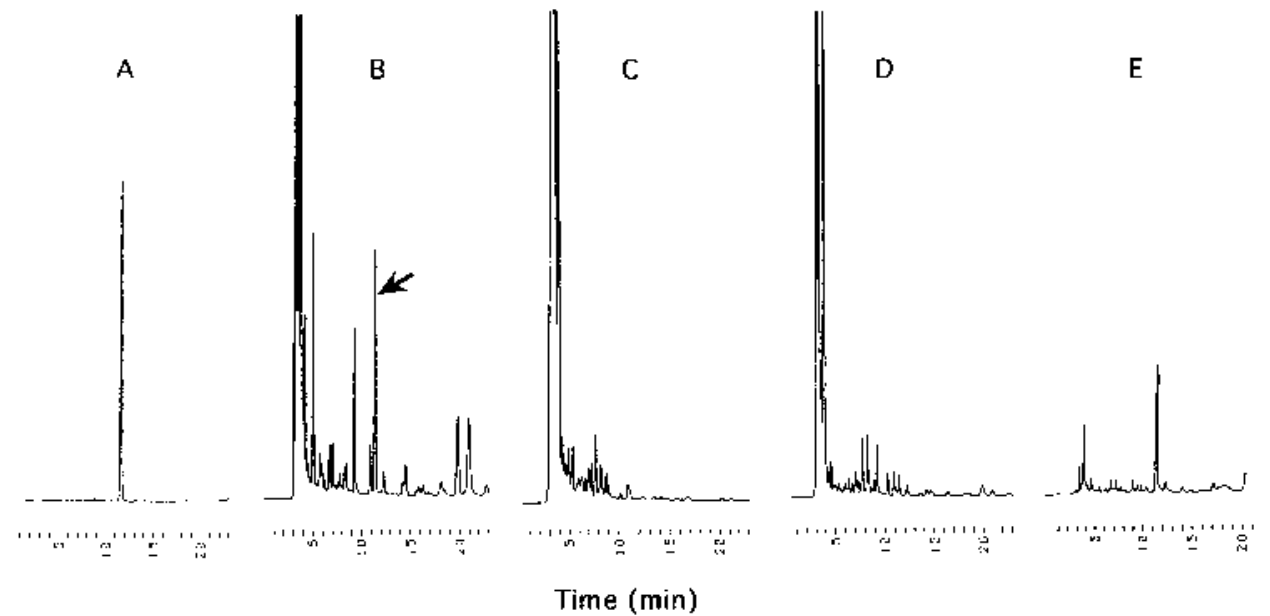

Fig. 2. High Performance Liquid Chromatograms of Pyrraline

A; pyrraline standard $(11.25 \mu \mathrm{M})$, B; urinary pyrraline determined by the proposed method. The arrow indicates pyrraline identified by mass spectrometer, as described in Fig. 3 B, C; urine sample flowed out from the cartridge for pretreatment, D; washing solvent that passed through the cartridge after loading of human urine to it, E; alkaline-hydrolyzed urine sample of (B). An equal volume of $4 \mathrm{M} \mathrm{LiOH}$ was added to the urine sample, which was then heated at $108^{\circ} \mathrm{C}$ for $18 \mathrm{~h}$ in a screw-capped tube. The alkaline-hydrolyzed urine sample, the concentration of which was $1 / 2$ of sample B, was then applied to the HPLC system. 


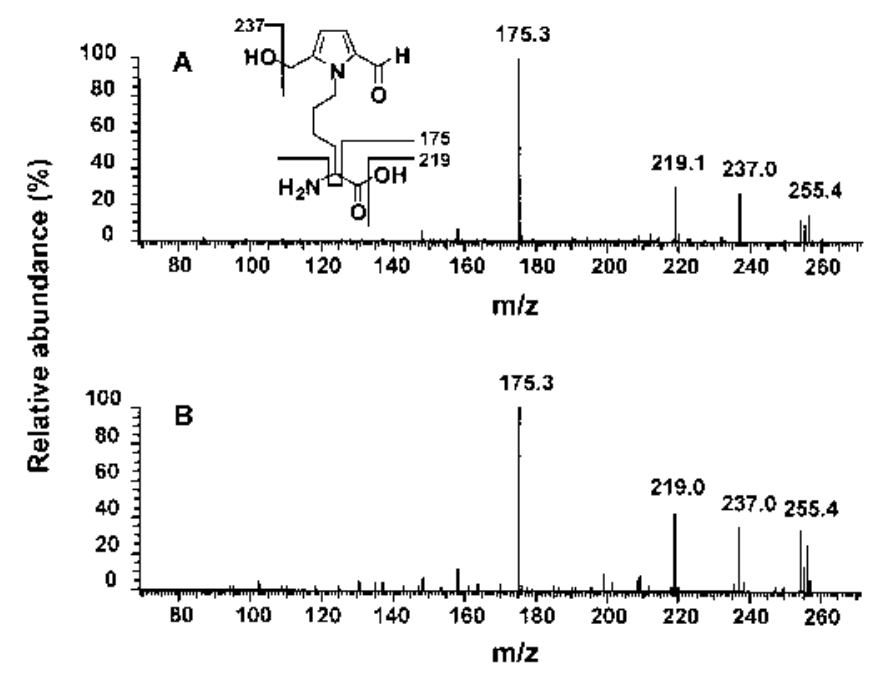

Fig. 3. Mass Spectra for the HPLC-MS/MS Analysis of Pyrraline Standard (A) and Urinary Pyrraline (B)

Chromatographically separated pyrraline was introduced directly to the quadrupole ion trap MS/MS system operating in the APCI positive-ion mode. Other conditions are expatiated in Materials and Methods.

time at about $11.5 \mathrm{~min}$ (Figs. $2 \mathrm{~A}$ and $2 \mathrm{~B}$, respectively). When urine sample was passed through the Oasis ${ }^{\mathrm{TM}}$ HLB cartridge, no pyrraline remained in the sample (Fig. 2C). Moreover, only a small amount of pyrraline was present in the washing solvent that flowed out from the cartridge (Fig. 2D).

In order to identify the chromatographically separated urinary pyrraline, we performed APCI mass spectrometry of the peak. The ion trap MS/MS spectrum for the pyrraline standard shown in Fig. 3A reveals an ion for $[\mathrm{M}+\mathrm{H}]^{+}$at $m / z=255$ and daughter ions corresponding to fragmentation. A pattern characteristic for standard pyrraline was obtained for urinary pyrraline, as shown in Fig. 3B, confirming that the peak is that for pyrraline. The mass spectrum for urinary pyrraline obtained by the MS/MS full scan mode simultaneously indicated the absence of contaminants (data not shown).

The plots of the peak areas obtained with the standard pyrraline solutions against concentration gave a linear relationship, $y=502+8844 x[y$ and $x$ refer to the peak area $(\mu \mathrm{V} \cdot \mathrm{s})$ and the molar concentration $(\mu \mathrm{M})$ of pyrraline, respectively], correlation coefficient $r=0.999,100 \mathrm{nM}-$ $100 \mu \mathrm{M}$. A recovery test was performed to confirm the precision of this proposed method. A known concentration of pyrraline $(10 \mu \mathrm{M})$ was added to urine samples from 6 healthy adults, and the mean recovery rate of pyrraline was $97.2 \pm$ $3.3 \%$. These results suggest that other components in the urine samples from different subjects did not affect the quantitative determination of pyrraline.

In order to estimate the amount of peptide-bound pyrraline in urine, alkaline hydrolysis was performed before pretreatment of the sample and analysis. The stability of pyrraline toward alkaline hydrolysis was confirmed previously. ${ }^{7,17)}$ Very small amounts of the peptide-bound form of pyrraline were present in the urine, because alkaline hydrolysis did not affect the recovery of pyrraline, as shown in Figs. 2B and 2E. Therefore, a hydrolysis process is not required for quantification of total urinary pyrraline.

Figure 4 shows the urinary excretion levels of pyrraline in

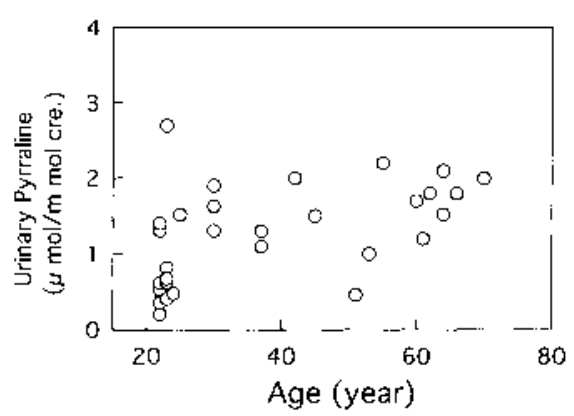

Fig. 4. Urinary Excretion Levels of Pyrraline in Healthy Volunteers

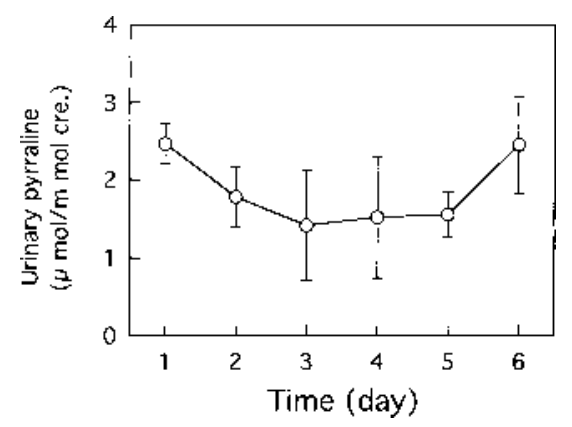

Fig. 5. Day to Day Variation in Urinary Excretion Level of Pyrraline

Urine samples from one healthy young female of 22 years old were investigated for $6 \mathrm{~d}, 3$ times a day; morning, noon and evening. Each point and bar indicates the mean \pm standard deviation of 3 determinations per day.

control subjects. These levels were expressed as $\mu \mathrm{mol} / \mathrm{mmol}$ creatinine, which corrects for urine dilution and body mass. The urinary pyrraline excretion levels appeared to slightly increase with age, but no significant correlation was observed. The mean excretion level of these control subjects was $1.42 \pm 0.65 \mu \mathrm{mol} / \mathrm{mmol}$ creatinine, which is similar to that reported by Portero-Otin et al. ${ }^{15)}$ Formation of AGEs, particularly that of pentosidine, via the Maillard reaction is known to increase with age. ${ }^{3,18,19)}$ However, pyrraline formation in collagen was not affected by aging. ${ }^{20)}$ It is therefore considered that urinary pyrraline in healthy subjects aged over 20 years old may remain at a certain level. The day to day variation in urinary pyrraline level was also investigated. As shown in Fig. 5, the amount of pyrraline excreted remains at a certain level, i.e. below $3 \mu \mathrm{mol} / \mathrm{mmol}$ creatinine, and the variation in the excretion level was considered insignificant.

To date, quantitative determination of pyrraline has usually been performed by HPLC, mass spectrometric, and immunologic methods. ${ }^{8-11,20,21)}$ For the HPLC method, a number of procedures have been reported but they had problems that remained to be solved. ${ }^{15,16)}$ For example, direct infusion of a urine sample to an HPLC equipment, which shortens the life span of the column, requires collection of the pyrraline fraction in the first chromatography and performance of re-chromatography in a second system. Such methods complicate the chromatographic procedure and are time-consuming. The proposed method described here eliminates these problems by using a pretreatment procedure. Moreover, application of the new procedure presented here to the determination of pyrraline levels in serum and other biological fluids should help obtain information on the role of pyrraline in the body more easily, rapidly, and accurately. 
Acknowledgment This work was supported in part by a grant for private universities provided by the Japan Private School Promotion Foundation.

\section{REFERENCES}

1) Nakayama T., Hayase F., Kato H., Agric. Biol. Chem., 44, 1201-1202 (1980).

2) Brownlee M., Cerami A., Vlassara H., New Engl. J. Med., 318, 13151321 (1988).

3) Sell D. R., Monnier V. M., J. Biol. Chem., 264, 21597-21602 (1989).

4) Uchiyama A., Ohishi T., Takahashi M., Kushida K., Inoue T., Fujie M., Horiuchi K., J. Biochem. (Tokyo), 110, 714-718 (1991).

5) Grandhee S. K., Monnier V. M., J. Biol. Chem., 266, 11649-11653 (1991).

6) Dyer D. G., Blackledge J. A., Thorpe S. R., Baynes J. W., J. Biol. Chem., 266, 11654-11660 (1991).

7) Hayase F., Nagaraj R. H., Miyata S., Njoroge F. G., Monnier V. M., J. Biol. Chem., 263, 3758-3764 (1989).

8) Miyata S., Monnier V. M., J. Clin. Invest., 89, 1102-1112 (1992).

9) Odani H., Shinzato T., Matsumoto Y., Takai I., Nakai S., Miwa M., Iwayama N., Amano I., Maeda K., Biochem. Biophys. Res. Commun., 224, 237-241 (1996).

10) Kato H., Shin D. B., Hayase F., Agric. Biol. Chem., 51, 2009-2011
(1987).

11) Shin D. B., Hayase F., Kato H., Agric. Biol. Chem., 52, 1451-1458 (1988).

12) Knecht K. J., Feather M. S., Baynes J. W., Arch. Biochem. Biophys., 294, 130-137 (1992).

13) Yamada H., Miyata S., Iigaki N., Yatabe H., Miyauchi Y., Ohara T., Sakai M., Shoda H., Oimomi M., Kasuga M., J. Biol. Chem., 269, 20275-20280 (1994).

14) Miyata T., Ueda Y., Yamada Y., Izuhara Y., Wada T., Jadoul M., Saito A., Kurokawa K., van Ypersele de Strihou C., J. Am. Soc. Nephrol., 9, 2349-2356 (1998).

15) Portero-Otin M., Pamplona R., Bellmunt M. J., Bergua M., Nagaraj R. H., Prat J., Life Sci., 60, 279-287 (1997).

16) Portero-Otin M., Pamplona R., Bellmunt M. J., Bergua M., Prat J., Eur. J. Clin. Invest., 27, 767-773 (1997).

17) Nagaraj R. H., Sady C., FEBS Lett., 382, 234-238 (1996).

18) Takahashi M., Hoshino H., Kushida K., Inoue T., Anal. Biochem., 232, 158 -162 (1995).

19) Takahashi M., Kushida K., Ohishi T., Kawana K., Hoshino H., Uchiyama A., Inoue T., Arthritis Rheum., 37, 724-728 (1994).

20) Portero-Otin M., Nagaraj R. H., Monnier V. M., Biochim. Biophys. Acta, 1247, 74-80 (1995).

21) Makita Z., Vlassara H., Cerami A., Bucala R., J. Biol. Chem., 267, $5133-5138$ (1992) 